\title{
The Effects of Pilates and Aerobic Exercise on Blood Pressure, Heart Rates, and Blood Serum Lipids in Sedentary Females
}

\author{
Semra Çetin ${ }^{1}$ Cuma Ece ${ }^{1}$, Murat Şen ${ }^{1}$, H. Nedim Çetin ${ }^{2}$, Alaeddin Aydoğan ${ }^{2}$ \\ ${ }^{1}$ Faculty of Sport Sciences, Sakarya University, Sakarya, Turkey \\ ${ }^{2}$ Faculty of Sport Sciences, Lokman Hekim University, Ankara, Turkey \\ Correspondence: H.Nedim Çetin, Faculty of Sport Sciences, Lokman Hekim University, Ankara, Turkey.
}

Received: February 20, 2019

Accepted: March 6, 2019 Online Published: March 24, 2019

doi:10.11114/jets.v7i4.4077

URL: https://doi.org/10.11114/jets.v7i4.4077

\begin{abstract}
The aim of this study was to determinate the effects of 12 weeks pilates and aerobic exercise on blood pressure, heart rates, and blood serum lipids in sedentary females. 18 sedentary women with an average age of 45.52 years, height of $161.14 \mathrm{~cm}$ and weight of $72.5 \mathrm{~kg}$ have been selected and put through a plates and aerobic exercise programmer one hour a day for three days a week. Exercises in each training session were arranged in such a way as to make each woman's heart rate to reach a level of 130-140 a minute. The waist and hip circumferences were measured using a measuring tape. BMI and waist hip ratio were calculated by standard formulas. Triglyceride (TG), Total Cholesterol (TC), High density lipoprotein (HDL-C) and Low density lipoprotein (LDL-C) levels were determined by Hitachi 717 auto analyzer. Analysis was performed on SPSS 21 version. Paired-t tests were done statistical analysis. Body Weight found before 12 weeks plates and aerobic exercise $72.5 \mathrm{~kg}$ and after $63.8 \mathrm{~kg}$. At the end of the 12 weeks exercise program, a decrease of \%12.00 in body weight, \%14.03 in systolic, \%3.96 in diastolic, \%13.85 in cholesterol, \%25.30 in Triglyceride, and \%22.33 in LDL-C have been registered. However, there were increases of \%16.34 in HDL-C. The effects of aerobic exercise is on Triglyceride, HDL-C, LDL-C and total cholesterol since $(\mathrm{p}<0.01)$. At the end of the 12 weeks exercise program, a decrease $\% 13.34$ hip circumference and $\% 4.19$ waist circumference. Waist to hip ratio found before 12 weeks plates and aerobic exercise $0.87 \mathrm{~cm}$ and after $0.78 \mathrm{~cm}$. The effects of aerobic exercise is on Body weight, Systolic blood pressure, Heart rate, hip and waist circumference since $(\mathrm{p}<0.05 ; \mathrm{p}<0.01)$. In this study, together Pilates and aerobic exercise was effective in sedentary women with initially high total cholesterol, triglyceride, and Low density lipoprotein levels. At end of the cycles of 12 weeks Pilates and aerobic exercises, has a positive effect of waist to hip ratio, blood pressure, and heart beats in sedentary females. Risk of heart and vascular disease is reduced. Pilates and aerobic exercises are recommended for decrease risk.
\end{abstract}

Keywords: waist hip ratio, exercise, sedentary women

\section{Introduction}

Human is dynamic existence and should move to survive. If the movement is performed with some rules and discipline, in order to purpose, ensure our physical and physiological health. It is clear that physical inactivity is a major cause of premature mortality and morbidity from chronic diseases (Warburton et al, 2006; World Health Organization, 2004). Modern life physically motionless effects every aged of people. The problems such as; hypertension, obesity, muscle atrophy, postural disorder and inadequate cardiovascular system caused by lack of exercise and sedentary life style or harmful habits such as smoking (Imamoğlu et al., 2017). Cardiovascular disease (CVD) in women is the leading cause of mortality in the United States, and less than optimal lipid and lipoprotein levels are major risk factors for CVD (George at al., 2004). There is consolidated evidence that physical activity exerts beneficial effects on several chronic conditions and longevity, on the basis of its proposed biological effects, especially on lipid profiles (Lippi et al., 2006). Aerobic exercise is efficacious for increasing high density lipoprotein and decreasing total cholesterol, low density lipoprotein, and triglyceride in women (George at al., 2004). High-intensity aerobic training results in improvement in high-density lipoprotein cholesterol (Tambalis et al., 2008). Being overweight is related to the development of many diseases like diabetes, coronary heart disease, psychological disorders, kidney disease, hypertension, stroke, lung disease and back and foot problems (Öztürk and Bavl1, 2017). Cardiovascular disease is the leading cause of death worldwide. Low blood levels of high-density lipoprotein cholesterol are an independent risk factor for cardiovascular 
disease (Franceschini, 2001). Cross-sectional data provide strong evidence that people who are more physically active have higher High density lipoprotein levels. Thus, the value of regular aerobic exercise in increasing serum high density lipoprotein level and in reducing the risk of cardiovascular disease has received widespread acceptance. In contrast, results of aerobic exercise studies vary considerably, depending on the exercise program and characteristics of subjects at baseline (Imamoğlu et al., 2014). Comparisons between intensities of aerobic exercise programs resulted in favorable effects only for high intensity. The most frequently observed alteration was an increase in the high-density lipoprotein cholesterol, whereas reductions in triglycerides, total cholesterol, and low-density lipoprotein cholesterol appeared less often (Tambalis et al., 2008). Various researchers suggest that aerobic group exercise classes e.g. cycling, and zumba taken regularly are effective in controlling weight, blood pressure, and body composition (Delextrat et al., 2016; Faulkner et al., 2015). Acute short term physiological responses to aerobic exercise include increased heart rate, blood pressure, breathing rate, and tidal volume. Long-term aerobic exercise participation is proven to increase the amount of maximal oxygen consumption during intense aerobic exercise, lower resting blood pressure and heart rate, lower body fat mass, and increase muscle mass, while low levels of cardiovascular fitness are associated with increased risk of premature death (Ehrman et al., 2009). Hence, aerobic exercise plays an important role in decreasing the risk of cardiovascular disease, pulmonary diseases, and metabolic diseases (Pescatello et al., 2013). Both aerobic dance and zumba incorporate large muscle groups for movement during class for aerobic endurance, strength training and flexibility. Exercise will also help to regulate hormonal changes during menopause and it reduces back pain, limit body weight gain and fat retention post pregnancy (Ehrman et al., 2009; Imamoğlu, 2017). To get rid of all these adverse circumstances in order to make fit and healthy organism is needed to important activities increasingly, such as sport for a healthy life, fitness, aerobics, jogging, and pilates etc. (Gullu et al., 2013;Çetinkaya and Imamoğlu, 2018).

Studies have shown that Pilates reduces the risk of heart diseases, prevents osteoporosis, gives the body a good form and develops balance, flexibility and strength (Solomon, 2003). When literature was reviewed about effects of exercise on plasma lipids, lipoproteins, blood pressure and heart rates, results were indicated that moderate and low intensity exercise, if its performed adequate time period cause decrease in body weight and body fat ratio, additionally decrease serum total cholesterol level. Though pilates is an exercise with lower intensity compared to other aerobic and dance exercises, the concentration, control, focus, flowing movement rhythm, certainty of movement and breathing techniques during positions play a very important role in a healthy body (Öztürk and Bavll, 2017). Several studies revealed improved fitness, weight loss, reductions in cholesterol levels or inflammatory markers in women at risk after various forms of aerobic dancing Shimamoto, including "aerobics", "step aerobics" and "cultural dances" ( Sharma et al., 2017). The Pilates exercises has been used since the beginning of the 20th century as a form of exercise that was devised to improve strength, flexibility, and muscular control, particularly of the muscles of the lumbar and pelvic regions, which are essential for activities of daily living (Granacher at al., 2013). Recent meta-analysis articles suggest that Pilates can improve different physical functional abilities in older individuals (Bullo et al., 2015; Barker et al., 2015; Campos et al., 2016). In the literature, there are many studies involving pilates training (Barker et al., 2015). The Pilates exercises is an exercise and physical movement system that has become popular all over the world and has been used in both rehabilitation and fitness. Öztürk and Bavlı (2017) in a study, it is found to say that eight weeks of step aerobics and pilates training has positive and similar effects on structural, biomotor and psychological variables of sedentary women.

The lipid and lipoprotein abnormalities play a major role in the development and progression of coronary artery disease. Benefits include improved serum lipid profiles, blood pressure and inflammatory markers as well as reduced risk of stroke, acute coronary syndrome and overall cardiovascular mortality (Skoumas et al., 2003; Baydil, 2013). The importance of regular physical activity is emphasized in order to maintain a healthy life and to have both preventive and healing effects for many diseases and injuries (Brown et al., 2012; Yilmaz et al., 2017).

The combination of pilates and aerobic exercises is thought to have a positive effect. The purpose of this study was to determinate the effects of 12 weeks pilates and aerobic exercise on waist to hip ratio, blood pressure, heart rates, and blood serum lipids in sedentary females.

\section{Method}

\subsection{The Study Group}

Eighteen healthy sedentary female whose average age is 45.52 years participate this study as a subject. Females are volunteered to participate in this study. All the participants were informed of the purpose of the study.

\subsection{Training Program}

Plates and Aerobic groups participate the training during 12 weeks period with one hour training session 3 days in a week period. The study was conducted in two parts. In training was 30 minute Pilates and 30 minute aerobic exercise. Intensity of training increased slightly and heart rates were 130-140 beats in minute at the end of exercise. We also excluded studies of subjects having specific medical problems in which treatments such as with diet or drugs would 
influence the effect of exercise (history of cancer, hemodialysis treatment, and coronary heart disease). At the beginning of the study, blood samples were taken from all subjects before breakfast in the morning. High density lipoprotein (HDL-C), low density lipoprotein (LDL-C), total cholesterol (TC) and triglyceride (TG) levels were determined these blood samples. Triglyceride, total cholesterol, high density lipoprotein and low density lipoprotein levels were determined by hitachi 717 auto analyzer. The blood pressure and heart beats were measured by digital blood pressure meters ALP K2 777. At the end of 12 weeks period all measurements were repeated. The waist and hip circumferences were measured using a measuring tape. BMI and waist hip ratio were calculated by standard formulas. $(\mathrm{BMI})=$ Body Weight $(\mathrm{kg}) /$ Height $(\mathrm{m})^{2}=\left(\mathrm{kg} / \mathrm{m}^{2}\right)$. Waist to hip ratio= Hip circumference/ Waist circumference.

\subsection{Statistics}

Analysis was performed on SPSS 21 version. Shapiro Wilk test was used to evaluate the normality of parameters. The difference between pre-test, post-test results was determined paired $t$ test. The significance level was determined as $\mathrm{p}<0.05$ and $\mathrm{p}<0.001$. For all the values, mean and standard deviation were used.

\section{Results}

In table 1 , shown anthropometrical parameters of females before and after pilates and aerobic exercise participate. In table 2, shown alteration rates of blood parameters. In table 3, shown blood pressure and heart rates alteration.

Table 1. Anthropometrical parameters of sedentary females before and after pilates and aerobic exercise $(n=18)$

\begin{tabular}{lcccc}
\hline Variables & Before Exercise & After Exercise & $\begin{array}{c}\text { Decrease and } \\
\text { Increase }\end{array}$ & $\mathrm{t}$ \\
\hline Age $($ year$)$ & 45.52 & - & - & - \\
\hline Height $(\mathrm{cm})$ & 161.14 & - & -12.00 & $2.21^{*}$ \\
\hline Body Weight $(\mathrm{kg})$ & 72.5 & 63.8 & -12.01 & $2.12^{*}$ \\
\hline Body mass index $\left(\mathrm{kg} / \mathrm{m}^{2}\right)$ & 28.32 & 24.92 & -13.34 & $3.63^{* *}$ \\
\hline Hip circumference $(\mathrm{cm})$ & 90.15 & 78.12 & -4.19 & $2.92^{*}$ \\
\hline Waist circumference $(\mathrm{cm})$ & 104.18 & 99.82 & - & - \\
\hline Waist to hip ratio & 0.87 & 0.78 & & \\
\hline
\end{tabular}

$* \mathrm{p}<0.05 \quad * * \mathrm{p}<0.001$

Table 2. Percent change in blood parameters of sedentary female

\begin{tabular}{|c|c|c|c|c|c|}
\hline $\mathrm{Mg} / 100$ & & Mean & Std. Deviation & $\begin{array}{c}\text { Decrease } \\
\text { and Increase }\end{array}$ & $\mathrm{t}$ \\
\hline \multirow{2}{*}{ Cholesterol (mg/cc) } & Before Exercise & 196.77 & 34.07 & \multirow{2}{*}{-13.83} & \multirow{2}{*}{$7.12 * *$} \\
\hline & After Exercise & 169.55 & 32.62 & & \\
\hline \multirow{2}{*}{ Triglyceride $(\mathrm{mg} / \mathrm{cc})$} & Before Exercise & 109.82 & 35.19 & \multirow{2}{*}{-25.30} & \multirow{2}{*}{$5.33 * *$} \\
\hline & After Exercise & 82.03 & 20.72 & & \\
\hline \multirow{2}{*}{ HDL-C (mg/cc) } & Before Exercise & 39.14 & 4.28 & \multirow{2}{*}{22.33} & \multirow{2}{*}{$-4.82 * *$} \\
\hline & After Exercise & 47.88 & 12.73 & & \\
\hline \multirow{2}{*}{ LDL-C (mg/cc) } & Before Exercise & 117.79 & 32.55 & \multirow{2}{*}{-21.69} & \multirow{2}{*}{$5.64 * *$} \\
\hline & After Exercise & 92.24 & 20.78 & & \\
\hline
\end{tabular}

$* * \mathrm{p}<0.001$

Table 3. Percent change in blood pressure and heart rates of sedentary females

\begin{tabular}{|c|c|c|c|c|}
\hline Variables & Before Exercise & After Exercise & $\begin{array}{c}\text { Decrease and Increase } \\
(\%)\end{array}$ & $\mathrm{t}$ \\
\hline $\begin{array}{l}\text { Systolic blood pressure } \\
(\mathrm{mmHg})\end{array}$ & 127.12 & 109.28 & -14.03 & $-2,23 *$ \\
\hline $\begin{array}{l}\text { Diastolic blood pressure } \\
(\mathrm{mmHg})\end{array}$ & 76.50 & 73.47 & -3.96 & $-1,02$ \\
\hline Heart Rate & 88.40 & 76.32 & -13.67 & $-2,14 *$ \\
\hline
\end{tabular}

$* \bar{p}<0.05$

\section{Discussion}

Physical activity and exercise habit contribute to prevent obesity, cardiovascular diseases, diabetes and some other chronic diseases and to lead a healthy lifestyle. Exercise and physical activity are at the top of the treatment options list to prevent hyperlipidemia, one of the significant risk factors for cardiovascular diseases (Demirel et al., 2018). When literature was reviewed about effects of exercise on plasma lipids, lipoproteins, blood pressure and heart rates, results were indicated that moderate and low intensity exercise, if its performed adequate time period decrease in body weight and body fat ratio, additionally decrease serum TC level. In the most studies, it has been noticed that regular exercise affects levels of lipid and lipoprotein positively and it is salutary for the heart. It has been identified that regular exercise reduces total blood cholesterol, serum triglycerides and LDL-C and increases high-density lipoprotein cholesterol 
HDL-C (Imamoğlu et al., 2017). Previous epidemiologic studies have demonstrated an increased risk of CHD and cardiovascular death with an increase in BMI (Garry and McShane, 2001).

The effect of BMI was presented in four categories according to World Health Organization

Recommendations: $<18.5 \mathrm{~kg} / \mathrm{m}^{2}$ (lean); $18.5-24.9 \mathrm{~kg} / \mathrm{m}^{2}$ (normal weight); $25-29.9 \mathrm{~kg} / \mathrm{m}^{2}$ (overweight) and $\geq 30 \mathrm{~kg} / \mathrm{m}^{2}$. Another classification: BMI's categories (the underweight $\left[<18.5 \mathrm{~kg} / \mathrm{m}^{2}\right]$, low normal weight $\left[18.5-21.9 \mathrm{~kg} / \mathrm{m}^{2}\right]$, normal weight [22-23.9 kg/m ${ }^{2}$, the referent], overweight $\left[24-26.9 \mathrm{~kg} / \mathrm{m}^{2}\right]$, obese $1\left[27-29.9 \mathrm{~kg} / \mathrm{m}^{2}\right]$, and obese $2[\geq 30$ $\left.\mathrm{kg} / \mathrm{m}^{2}\right]$ ), (Hsu et al., 2018)). Multiple meta-regression analyses demonstrated that subjects with a body mass index less than 28 and total cholesterol level of $220 \mathrm{mg} / \mathrm{dL}$ or more experienced an approximately $2.1-\mathrm{mg} / \mathrm{dl}$ larger increase in HDL-C level than those with a body mass index of 28 or more and total cholesterol level less than $220 \mathrm{mg} / \mathrm{dl}$ (Kodama et al., 2007). Elevation in body mass index (BMI) has been associated with less favorable lipoprotein profiles. Elevation in the BMI has also been associated with an increased relative risk of CHD, as well as an increased mortality due to cardiovascular disease (Garry and McShane, 2001). In a study, players with BMI $>32 \mathrm{~kg} / \mathrm{m}^{2}$ had a relative risk of cardiovascular death that was 6.04 times that of the players with BMI $<28 \mathrm{~kg} / \mathrm{m}^{2}$ (Garry and McShane, 2001). There is a general understanding that the body mass index (BMI) in itself is a major determinant of mortality, although qualified by fat distribution for disease specific outcomes (Whitlock et al., 2009; Wang et al., 2005). Historically, this may not always have been so but evidence from large-scale cohorts in both Western and Asian countries generally indicate that a BMI of 22-25 kg/m² may be optimal for prevention of premature death (Heymsfield and Cefalu, 2013; Hsu et al., 2018). In this study, body weight found before 12 weeks plates and aerobic exercise $72.5 \mathrm{~kg}$ and after $63.8 \mathrm{~kg}$. There was a significant decrease in body weight and body mass index $(\mathrm{p}<0.05)$. In this study, found at the end of the 12 weeks plates and aerobic exercise program, a decrease of $12.00 \%$ in body weight and body mass index $12.01 \%$. As a result of exercises body mass indexes decreased to normal values.

The waist to hip ratio was calculated as waist girth divided by the hip girth. It is statistically proved that men with WHR (Waist to hip ratio) of more than 0.90 and women with 0.85 are at greatest risk of health complications (Annamna et al., 2012). Waist hip ratio above 0.85 for females are important indicators of lifestyle-related health problems throughout the world (Dubbert, 2002). Sedentary women with waist circumference of more than $85 \mathrm{~cm}$ had almost doubled mortality risk compared to active women with waist circumference below $80 \mathrm{~cm}$ (Annamna et al., 2012). Turgut et al. (2017) in a study, found at the end of three months of exercise, the decrease in waist and hip circumference measurements significant. In this study, found at the end of the 12 weeks plates and aerobic exercise program, a decrease $13.34 \%$ hip circumference and $4.19 \%$ waist circumference.WHR found before 12 weeks plates and aerobic exercise $0.87 \mathrm{~cm}$ and after $0.78 \mathrm{~cm}$. The effects of pilates and aerobic exercise is positive hip and waist circumference since $(\mathrm{p}<0.05 ; \mathrm{p}<0.01)$.

Several studies have shown that exercise can cause improving effect on serum HDL-C, LDL-C and TG (Boardley et al., 2007). Çiçek et al. (2017) found step-aerobic and core exercise showed significant changes of inflammatory and lipid markers with cardiac dimensions and had favorable effects on both left ventricular systolic function. Turgut et al., (2017) in a study, found there a significant difference in cholesterol, triglyceride, and HDL-C and LDL-C values between the first measurement and the second measurement after 3 months of exercise. Imamoğlu et al. (2017) in a study, regular aerobic and weight-lifting plus aerobic exercises have been shown to reduce the risk of cardiovascular disease in sedentary women and a significant improvement in systolic and diastolic blood pressures as well as reduction of cholesterol, triglyceride, and of LDL-C. Level of HDL-C was increased in both of exercise groups. Gullu et al. (2013) in a study, there were decreases in run-walk group on total cholesterol by $6.44 \%$, LDL-C by $7.93 \%$, and triglyceride by $17.75 \%$, but there was not any difference in value of HDL-C. There were decreases in step-aerobics group on total cholesterol by $10.54 \%$, LDL-C by $9.05 \%$, and triglyceride by $15.24 \%(\mathrm{p}<0.01$ and $\mathrm{p}<0.05)$, however, there was increasing in value of HDL-C by 5.64\% (p<0.05). Imamoğlu et al. (2002), Koca (2017) and Imamoğlu et al (2017) in on studies, found to effect short time exercise on serum lipids of sedentary females increase in HDL-C and to decrease in LDL-C, total cholesterol, and triglyceride. After 12 weeks pilates and aerobic exercise in this study decrease percentage of blood values of females, found $13.3 \%$ in Cholesterol quantity, $25.30 \%$ in triglyceride, and $21.69 \%$ in LDL-C. Also HDL-C increase is $22.33 \%$ (Table 2). This study was Imamoğlu et al. (2002 and 2017) and is compatible with the work of the Koca (2017). At the end they couldn't found significance, triglyceride, total cholesterol, LDL-C was decreased, HDL-C was increased $(\mathrm{p}<0.001)$. The average net increase in HDL-C level by exercise itself was but highly significant. Coronary heart disease risk decrease \%1.5 cause of to increase \%1 of HDL-C (Imamoğlu, 2014; Imamoğlu et al., 2017). In that case in this study, decrease of LDL-C and increases of HDL-C have decreased risk of Coronary Heart Disease. 
Most of studies about blood pressure and heart beat, claim that the exercise decreased the blood pressure and heart beat (Imamoğu, 2014). Imamoğlu et al. (2017) on studies, the systolic blood pressure was decreased in aerobic exercise. Diastolic blood pressure was decreased in aerobic exercise. Heart beat was decrease with aerobic exercise. Koca (2017) in a study founding similar result. In this study, the systolic blood pressure was decreased $14.03 \%$ in plates and aerobic exercise. Diastolic blood pressure was decreased $3.96 \%$ in plates and aerobic exercise. Heart beat was decrease $11.61 \%$ in plates and aerobic exercise. The decrease in systolic blood pressure and heart rate was found to be significant in this study $(\mathrm{p}<0.05)$. There was no significant change in diastolic blood pressure $(\mathrm{p}>0.05)$. Pilates and aerobic exercises may be recommended to reduce hypertension.

Both aerobic and anaerobic exercise can decrease total cholesterol, raise high-density lipoprotein cholesterol levels, and lower the TC/HDL-C ratio (Garry and McShane, 2001). A substantial increase in aerobic physical activity within the population might be recommended to reverse adverse lipid abnormalities, especially in subjects with a higher cardiovascular risk (Lippi et al., 2006). Regular aerobic exercise modestly increases HDL-C level. Exercise was more effective in subjects with initially high total cholesterol levels or high body mass index (Kodama et al., 2007). In this study found similar conclusion.

In this study, together pilates and aerobic exercise was effective in sedentary women with initially high total cholesterol, triglyceride, and low density lipoprotein levels. At end of the cycles of 12 weeks pilates and aerobic exercises, has a positive effect of waist to hip ratio, blood pressure, and heart beats in sedentary females. Risk of heart and vascular disease is reduced. Pilates and aerobic exercises are recommended for decrease risk.

\section{References}

Barker, A., Talevski, J., \& Bird, M. L. (2015). Effect of pilates exercise for improving balance and decreasing falls risk in older adults: a systematic review with meta-analysis. Physiotherapy, 101, 111-112. https://doi.org/10.1016/j.physio.2015.03.249

Baydil, B. (2013). Serum macro-micro element responses to acute maximal physical exercise. World Applied Sciences Journal, 23(7), 945-949.

Boardley, D., Fahlman, M., Topp, R., Morgan, A. L., \& McNevin, N. (2007). The impact of exercise training on blood lipids in older adults. The American Journal of Geriatric Cardiology, 16(1), 30-35. https://doi.org/10.1111/j.1076-7460.2007.05353.x

Brown, W. J., McLaughlin, D., Leung, J., McCaul, K. A., Flicker, L., Almeida, O. P., \& Dobson, A. J. (2012). Physical activity and all-cause mortality in older women and men. Br J Sports Med, 46(9), 664-668. https://doi.org/10.1136/bjsports-2011-090529

Bullo, V., Bergamin, M., Gobbo, S., Sieverdes, J. C., Zaccaria, M., Neunhaeuserer, D., \& Ermolao, A. (2015). The effects of Pilates exercise training on physical fitness and wellbeing in the elderly: a systematic review for future exercise prescription. Preventive Medicine, 75, 1-11. https://doi.org/10.1016/j.ypmed.2015.03.002

Campos, R. R., Dias, J. M., \& Pereira, L. M. (2016). Effect of the Pilates method on physical conditioning of healthy subjects: a systematic review and meta-analysis. J Sports Med Phys Fitness, 56, 864-873.

Çetinkaya G., \& Imamoğlu G. (2018). Investigation of the Effect of Plates-Aerobic Exercises on Body Composition and Body Image in Obesity Female, the Journal of International Social Research, 11(59), 1451-1456.

Çiçek, G., Imamoglu, O., Gullu, A., Celik, O., Ozcan, O., Gullu, E., \& Yamaner, F. (2017). The effect of exercises on left ventricular systolic and diastolic heart function in sedentary women: Step-aerobic vs core exercises. Journal of Exercise Science \& Fitness, 15(2), 70-75. https://doi.org/10.1016/j.jesf.2017.07.002

Delextrat, A. A., Warner, S., Graham, S., \& Neupert, E. (2016). An 8-Week exercise intervention based on zumba improves aerobic fitness and psychological well-being in healthy women. J Phys Act Health. 13(2), 131-9. https://doi.org/10.1123/jpah.2014-0535

Demirel, N., Özbay, S., \& Kaya F. (2018). The Effects of Aerobic and Anaerobic Training Programs Applied to Elite Wrestlers on Body Mass Index (BMI) and Blood Lipids, Journal of Education and Training Studies, 6(4), 58-62. https://doi.org/10.11114/jets.v6i4.3085

Dubbert, P. M., Carithers, T., \& Sumner, A. E. (2002). Obesity, physical inactivity, and risk for cardiovascular disease. Am. J Med Sci., 324, 116-126. https://doi.org/10.1097/00000441-200209000-00002

Ehrman, J. K., Gordon, P. M., Visich, P. S., \& Keteyian, S. J. (2009). Clinical Exercise Physiology, (2nd ed.). Champaign, IL: Human Kinetics.

Faulkner, S. H., Pugh, J. K., Hood, T. M., Menon, K., King, J. A., \& Nimmo, M. A. (2015). Group studio cycling; an 
effective intervention to improve cardio-metabolic health in overweight physically inactive adults. Journal of Fitness Research, 4(2), 16-25.

Franceschini, G. (2001). Epidemiologic evidence for high-density lipoprotein cholesterol as a risk factor for coronary artery disease. Am J Cardiol, 88(12), 9-13. https://doi.org/10.1016/S0002-9149(01)02146-4

Garry, J. P., \& McShane, J. J. (2001). Analysis of lipoproteins and body mass index in professional football players. Preventive cardiology, 4(3), 103-108. https://doi.org/10.1111/j.1520-037X.2001.00534.x

George, A., Kelley, Kristi, S., Kelley, Zung, \& Vu, Tran. (2004). Aerobic exercise and lipids and lipoproteins in women: a meta-analysis of randomized controlled trials. Journal of Women's Health, 13(10), 1148-1164 https://doi.org/10.1089/jwh.2004.13.1148

Granacher, U., Gollhofer, A., Hortobágyi, T., Kressig, R. W., \& Muehlbauer, T. (2013). The importance of trunk muscle strength for balance, functional performance, and fall prevention in seniors: a systematic review. Sports Medicine, 43(7), 627-641. https://doi.org/10.1007/s40279-013-0041-1

Gullu, E., Gullu, A., Cicek, G., Yamaner, F., Imamoglu, O., \& Gumusdag, H. (2013). The effects of aerobic exercises on cardiovascular risk factors of sedentary women. Int. J. Acad. Res, 5, 160-167. https://doi.org/10.7813/2075-4124.2013/5-3/A.23

Heymsfield, S. B., \& Cefalu, W. T. (2013). Does body mass index adequately convey a patient's mortality risk? Jama, 309(1), 87-88. https://doi.org/10.1001/jama.2012.185445

Hsu, C. C., Wahlqvist, M. L., Wu, I. C., Chang, Y. H., Chang, I. S., Tsai, Y. F., ... Hsiung, C. A. (2018). Cardiometabolic disorder reduces survival prospects more than suboptimal body mass index irrespective of age or gender: a longitudinal study of 377,929 adults in Taiwan. BMC Public Health, 18(1), 142. https://doi.org/10.1186/s12889-018-5038-0

Imamoglu, O. (2014). Acute effect of aerobic and anaerobic exercise on lipid levels. HealthMED, 8(1), 112-118.

Imamoğlu, O., Akyol, P., \& Satici, A. (2017). The effect of aerobic exercise and weight-lifting plus aerobic exercise on blood pressure and blood parameters in sedentary females. European Journal of Physical Education and Sport Science, 3(11), 194-206.

Koca F. (2017). The effect of 12 weeks aerobic exercise on blood pressure, heart rates and blood parameters in sedentary females, International Journal of Academic Research, Part A. Applied and Natural Sciences, 9(1), 5-7. https://doi.org/10.7813/2075-4124.2017/9-1/A.1

Kodama, S., Tanaka, S., Saito, K., Shu, M., Sone, Y., Onitake, F., ... \& Ohashi, Y. (2007). Effect of aerobic exercise training on serum levels of high-density lipoprotein cholesterol: a meta-analysis. Archives Of Internal Medicine, 167(10), 999-1008. https://doi.org/10.1001/archinte.167.10.999

Lippi, G., Schena, F., Salvagno, G. L., Montagnana, M., Ballestrieri, F., \& Guidi, G. C. (2006). Comparison of the lipid profile and lipoprotein (a) between sedentary and highly trained subjects. Clinical Chemistry and Laboratory Medicine (CCLM), 44(3), 322-326. https://doi.org/10.1515/CCLM.2006.056

Öztürk F., \& Bavlı Ö. (2017). Investigation of the Effects of Eight Weeks of Pilates and Step-Aerobic Exercises on Physical Performance and Self Esteem Scores of Females. International Journal of Science Culture and Sport, 5(2), 76-86. https://doi.org/10.14486/IntJSCS650

Pescatello, L. S., Riebe, D., \& Thompson, P. D. (Eds.). (2013). ACSM's guidelines for exercise testing and prescription. Lippincott Williams \& Wilkins.

Sharma, R., Suri, M., \& Saini, N. (2017). Physiological responses of zumba: an overview understanding the popular fitness trend, Indian journal of physical education. Sports and Applied Science, 7(4), 23-31.

Skoumas, J., Pitsavos, C., Panagiotakos, D. B., Chrysohoou, C., Zeimbekis, A., Papaioannou, I., \& Stefanadis, C. (2003). Physical activity, high density lipoprotein cholesterol and other lipids levels, in men and women from the ATTICA study. Lipids in Health and Disease, 2(1), 3. https://doi.org/10.1186/1476-511X-2-3

Solomon, L. (2003). Yogalates. 1st edition. London. Virgin Books Ltd. 2003, 25.

Tambalis, K., Panagiotakos, D. B., Kavouras, S. A., \& Sidossis, L. S. (2009). Responses of blood lipids to aerobic, resistance, and combined aerobic with resistance exercise training: a systematic review of current evidence. Angiology, 60(5), 614-632. https://doi.org/10.1177/0003319708324927

Turgut, M., Çınar, V., Akbulut, T., \& İmamoğlu, O. (2017). The effect of 3 month cardio bosu exercises on some motoric, physical and physiological parameters in sedentary women. International Scientific Conference, Sports, 
Education Culture, Book of Abstracts, ISSN 2457, Culture-Interdisciplinary Approaches in Scientific Research, 103.

Wang, Y., Rimm, E. B., Stampfer, M. J., Willett, W. C., \& Hu, F. B. (2005). Comparison of abdominal adiposity and overall obesity in predicting risk of type 2 diabetes among men. The American Journal of Clinical Nutrition, 81(3), 555-563. https://doi.org/10.1093/ajcn/81.3.555

Warburton, D. E., Nicol, C. W., \& Bredin, S. S. (2006). Health benefits of physical activity: the evidence. Cmaj, 174(6), 801-809. https://doi.org/10.1503/cmaj.051351

World Health Organization. (2004). WHO global strategy on diet, physical activity and health. Food Nutr Bull, 25(3), 292-302. https://doi.org/10.1177/156482650402500310

Yilmaz, A. K., Menderes, K., Mayda, H., Gul, C., \& Yalcin, T. (2017). Analysis of Q angle values of female athletes from different branches. Ovidius University Annals, Series Physical Education and Sport/Science, Movement and Health, 17(2), 141-147.

\section{Copyrights}

Copyright for this article is retained by the author(s), with first publication rights granted to the journal.

This is an open-access article distributed under the terms and conditions of the Creative Commons Attribution license which permits unrestricted use, distribution, and reproduction in any medium, provided the original work is properly cited. 